\title{
Von der Leiche zum Roboter - warum die ETHs sich täuschen
}

\section{Hans Stalder}

Prof. Dr. med., Mitglied der Redaktion

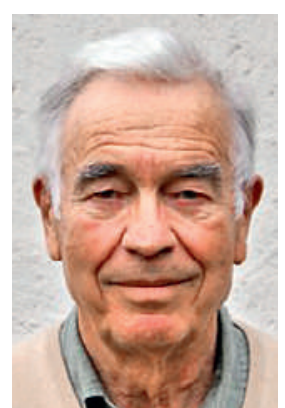

Als ich studierte, gab es nur zwei propädeutische Examen und ein eidgenössisches Examen am Ende des Studiums. Das erste Prope umfasste noch Zoologie und Botanik, während im zweiten die Anatomie eine wichtige Rolle spielte. Der erste «Mensch», dem man begegnete, war eine Leiche im Autopsiesaal. Nach jedem Examen war man erleichtert, "alles vergessen" zu können, was man gelernt hatte. Seither wurde das Medizinstudium reformiert und die Lernziele wurden genauer definiert. Es muss das gelernt werden, was in der Medizin wichtig ist! So wurden Zoologie und Botanik fallengelassen und die Anatomie verlor ihre Vorherrschaft an die Biologie, die Epidemiologie, die Ethik und weitere Humanwissenschaften. In gewissen Fakultäten, wie z.B. in Genf, wurde das Studium vollständig erneuert: In den präklinischen Jahren steht das problemorientierte Lernen im Vordergrund, während in den klinischen Praxisjahren das lösungsorientierte Lernen Priorität hat. Die Ausbildung erfolgt nicht mehr nach Disziplinen, sondern integrierend und umfassend. Eine möglichst frühe klinische Ausrichtung (in Genf im zweiten Studienjahr) wurde eingeführt: Der erste «Kranke» ist nicht mehr eine Leiche, sondern ein Patient im Spital oder in der Praxis eines Allgemeinmediziners. Die Studierenden sollen von der Bedeutung der Grundwissenschaften überzeugt werden, indem sie ihre klinische Anwendung kennenlernen. Die Einführung des Bologna-Systems mit Bachelor und Master hat dieses Konzept kaum beeinflusst: Das Medizinstudium bleibt eine Einheit vom 1. bis zum 6. Jahr. Das soll jedoch jetzt geändert werden: Die Eidgenössische Technische Hochschule (ETH) in Zürich schlägt vor, einen Bachelor in Medizin auf der Basis der MINT einzuführen: Mathematik, Informatik, Naturwissenschaften und Technik. Die ETH Lausanne hat ebenfalls ihr Interesse signalisiert.

Meiner Meinung nach gibt es mehrere Gründe, die belegen, dass dieser Vorschlag nicht gut durchdacht ist:

- «Dieser neue Studiengang entspricht vollumfänglich den Bedürfnissen des Standorts Nordwestschweiz mit seiner Life-Science-Industrie.» So äusserte sich die Rektorin der Universität Basel (NZZ, 12.9.2015). Das Medizinstudium darf sich nicht nach den Bedürfnissen der Industrie ausrichten, sondern nach denen der Bevölkerung! Und heutzutage müssen dringend praktizierende Ärzte ausgebildet werden. Zu diesem Zweck will der Bundesrat 100 Millionen Franken bereitstellen.
- In der Schweiz besteht vor allem ein schwerwiegender Mangel an Hausärzten. Die Unterstützung des Medizinstudiums durch den Bundesrat müsste also vor allem diesen Mangel beheben. Mathematik, Informatik und Technik gehören nicht zu den wesentlichsten Bereichen eines Hausarztes.

- Dies scheint auch die Meinung des Schweizer Volkes zu sein. Mit der aussergewöhnlich deutlichen Annahme eines Verfassungsartikels zur Hausarztmedizin und eines weiteren Artikels zur Alternativmedizin drückte das Volk ganz klar seinen Wunsch aus, dass die Medizin nicht noch technik- und informatiklastiger werden soll!

- Die Interdisziplinarität zwischen präklinischen und klinischen Wissenschaften ist ein entscheidender Vorteil des reformierten Medizinstudiums. Ein Programm, wie es von der ETH vorgeschlagen wird, wäre ein Rückschritt.

- Eine frühzeitige Ausrichtung auf die Klinik kann eine ETH nicht sicherstellen; sie ist jedoch ein wichtiges Element, um der Demotivation der Studierenden entgegenzutreten.

- Der Engpass beim Medizinstudium besteht nicht auf Bachelor-Stufe, wo es relativ einfach ist, die Anzahl der Studierenden zu erhöhen. Was fehlt, sind klinische Praktikumsplätze mit Patienten, ohne die ein Medizinstudium nicht denkbar ist. Die 100 Millionen müssen deshalb der klinischen Ausbildung zugutekommen. Diese kann vor allem durch heutige, den Universitätsspitälern angeschlossene medizinische Fakultäten, eventuell auch durch neue Standorte - Fribourg, St. Gallen, Luzern, Tessin - angeboten und in Arztpraxen ambulant entwickelt werden.

Sicher spielen die Informatik und die Technik heute und auch in Zukunft eine wichtige Rolle in der Weiterentwicklung der Medizin (und der Industrie ...). Deshalb ist auch eine Ausbildung durch Experten dieser Bereiche wünschenswert. Meiner Meinung nach sollte dies jedoch während der Weiterbildung in der Form eines Master of Advanced Studies geschehen und könnte durchaus durch die ETHs vermittelt werden. Wir halten an einer frühzeitigen Einführung in die medizinische Praxis mit echten Patienten fest und wollen nicht, dass der erste «Mensch», dem der Student begegnet, ein Roboter ist. 